\title{
Pre- and post-processing filters for improvement of blood velocity estimation
}

\section{Schlaikjer, Malene; Jensen, Jørgen Arendt}

\section{Published in:}

Proceeding of Ultrasonics Symposium

Link to article, DOI:

10.1109/ULTSYM.2000.921615

Publication date:

2000

Document Version

Publisher's PDF, also known as Version of record

Link back to DTU Orbit

Citation $(A P A)$ :

Schlaikjer, M., \& Jensen, J. A. (2000). Pre- and post-processing filters for improvement of blood velocity estimation. In Proceeding of Ultrasonics Symposium (Vol. 2, pp. 1531-1536). IEEE.

https://doi.org/10.1109/ULTSYM.2000.921615

\section{General rights}

Copyright and moral rights for the publications made accessible in the public portal are retained by the authors and/or other copyright owners and it is a condition of accessing publications that users recognise and abide by the legal requirements associated with these rights.

- Users may download and print one copy of any publication from the public portal for the purpose of private study or research.

- You may not further distribute the material or use it for any profit-making activity or commercial gain

- You may freely distribute the URL identifying the publication in the public portal 


\title{
Pre- and post-processing filters for improvement of blood velocity estimation
}

\author{
Malene Schlaikjer and Jørgen Arendt Jensen \\ Center for Fast Ultrasound Imaging, Department of Information Technology, Bldg. 344, \\ Technical University of Denmark, DK-2800 Kgs. Lyngby, Denmark
}

\begin{abstract}
The standard deviation on the blood velocity estimates are influenced by measurement noise, velocity spread, and signal alteration introduced by de-noising and clutter filters. A noisy and non-smooth appearance of the velocity distribution is obtained, which is not consistent with the actual velocity in the vessels. Post-processing is beneficial to obtain an image that minimizes the variation, and present the important information to the clinicians.

Applying the theory of fluid mechanics introduces restrictions on the variations possible in a flow field. Neighboring estimates in time and space should be highly correlated, since transitions should occur smoothly. This idea is the basis of the algorithm developed in this study. From Bayesian image processing theory an a posteriori probability distribution for the velocity field is computed based on constraints on smoothness. An estimate of the velocity in a given point is computed by maximization of the probability, given prior knowledge of the original estimate in that position, and the estimates in the neighboring positions in time and space.

The method has been tested on simulated 2D RF-data resembling signals from the carotid artery with different signalto-noise ratios (SNR). The exact extent of the vessel and the true velocities are thereby known. Velocity estimates were obtained by employing Kasai's autocorrelator on the data. The post-processing filter was used on the computed $2 \mathrm{D}$ velocity map. An improvement of the RMS error in the range of 15-53\% was observed. For low SNRs the highest improvement was obtained. Visual inspection of the images show a high qualitative improvement. A more smooth profile has been obtained, which more closely resembles the true smooth profile. The same conclusion can be drawn after application of the filter to in-vivo data acquired with a dedicated sampling system.
\end{abstract}

A short discussion of new ideas to differentiate flowing blood from moving tissue surrounding the blood are included in this paper. Neural networks and statistical discriminators are of interest, if it is possible to determine features for the different signal components.

\section{Introduction}

Ultrasound is widely used to obtain estimates of the blood velocities in the cardiovascular system. The spatial velocity distribution and the magnitudes contain information of diagnostic value. The processing of the recorded RF-data goes through a number of steps: pre-processing to remove noise and clutter signals, estimation of the blood velocities, and post-processing to create the image to display. Clutter filters are used to separate the tissue and blood components in the RF-signals, as only the velocity of the blood is of interest in this case. As both tissue and blood are moving, a discrimination is needed to determine, which estimates to display. Ideas for methods for the discrimination are discussed shortly in Section 2. The pre-processing is followed by the actual estimation of the blood velocities. The computed estimates are encumbered with uncertainty due to measurement noise, velocity spread, and signal alteration introduced by de-noising and clutter filters. A noisy and non-smooth appearance of the velocity distribution is obtained. The level of noise varies over the image and between consecutive images. The human visual system is sensitive to these rapid variations. The observers focus therefore gets distracted from the important information. Post-processing is necessary to obtain an image that minimizes the variation, and a new post-processing approach based on optical flow theory is introduced in Section 3. In Section 4 results are presented.

\section{Pre-processing of RF-data}

One of the main problems in velocity imaging is to differentiate between the flowing blood and moving tissue surrounding the blood [1]. The signals from the two often overlap, and it is difficult to find the exact boundary separating the two. Often the velocity estimates overlaps the boundary wrongly indicating flow outside the vessel or the algorithms do not detect flow at the vessel boundaries. Better approaches for doing the separation are thus needed. Discriminators based on neural networks and statistics are of interest, if one can determine 
at set of features that clearly distinguish the different signal components. The features need be stable with respect to noise variations and vessels size. Investigation into this area have been initiated. Employment of a simple 2-layer feed forward network [2] was used as a discriminator. The RF-lines were split up in a number of segments, and the discrimination is carried out on each of these. The input to the network is the values for a set of features. In this study the features were the energy of the segments before and after echo-canceling with a high pass (HP) filter, and the variance between values at the same depth location in consecutive RF-lines before and after echo-canceling with HP-filter. A total of 4 inputs were used. The outputs are the probability of whether the segment under investigation carry blood information or not.

A statistical discriminator can be designed from the distributions of the feature values. Two probability distributions arise for each of the features. A discrimination is carried out by assigning the segment to the component type with the highest probability.

The top plot in Fig. 1 shows the results of a discrimination with a neural network with 4 inputs, 20 hidden neurons, and one output. The discriminator has been used on simulated data resembling the carotid artery (see Section 4). Several frames were evaluated, and the result shows the worst outcome. The true segment discrimination is shown in the bottom plot. A total of $98.3 \%$ of the segments are correctly discriminated. The procentage of correctly discriminated blood and tissue segments are $94.9 \%$ and $99.0 \%$ respectively. The discriminator works well on the tissue segments and the blood segments in the middle of the vessel. Unfortunately the transition from tissue to blood and vice versa is less well determined.

At this point the method need being compared with other methods. Then it will be possible to say, if an improvement with respect to discrimination is obtained by employing the neural network.

\section{Post-processing filter based on opti- cal flow theory}

As discussed in the introduction the computed blood velocity estimates are encumbered with uncertainty, and postprocessing is needed to minimize the noisy variation. The filters, which are currently applied, make use of the spatial and temporal correlation that exist among neighboring velocity estimates. The correlation property states, that the levels of the velocity in a spatial and temporal neighborhood are similar. In this context it usually means that the true velocity in a given location lies within the range of the velocities used in the filter. This is the case, when employing median filtering. No assumptions on the transitions from one velocity to another velocity in a neighboring spatial and temporal location
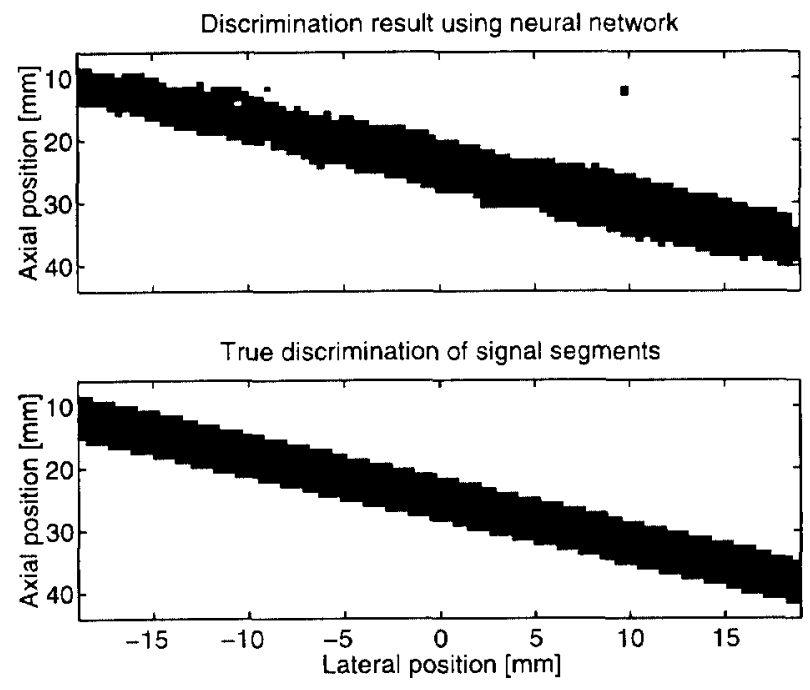

Figure 1: Discrimination result from employing a neural network. Segments with blood velocity information is marked with black.

are given. A filter, which employs the spatial and temporal correlation and introduces assumptions on the transitions, is introduced in this study.

The mechanics of fluid motion introduces restrictions on the possible flow patterns for blood. The velocity is a function of time and spatial location. The transition from one velocity to another in a neighboring point in time and space must be a continuous and smooth function. The smoothness property is the link to introduce restrictions on the variations possible between neighboring velocity estimates in time and space. A filter, which incorporates the smoothness property, can be derived from statistics. The filter computes an estimate of the true velocities $\vec{V}^{c}=\left\{\vec{v}^{c}\left(x_{1}, z_{1}, t\right), \ldots, \vec{v}^{c}\left(x_{N}, z_{M}, t\right)\right\}$ in the image, where $x_{i}$ and $z_{j}$ indicates the spatial location in the image, and $t$ is time. The available information for the filter is the estimated velocities, $\vec{V}^{e}=\left\{\vec{v}^{e}\left(x_{1}, z_{1}\right), \ldots, \vec{v}^{e}\left(x_{N}, z_{M}\right)\right\}$, for the present and previous image, and the smoothness property.

The filter is designed by employing Bayesian theory [3], where a conditional, posterior probability distribution, $p\left(\vec{V}^{c} \mid \vec{V}^{e}\right)$, for the true velocities can be obtained by:

$$
p\left(\vec{V}^{c} \mid \vec{V}^{e}\right)=\frac{p\left(\vec{V}^{c}\right) p\left(\vec{V}^{e} \mid \vec{V}^{c}\right)}{p\left(\vec{V}^{e}\right)}
$$

$p\left(\vec{V}^{c}\right)$ is the a priori probability distribution, and $p\left(\vec{V}^{e} \mid \vec{V}^{c}\right)$ is the model specific probability. $p\left(\vec{V}^{e}\right)$ is the probability distribution of the estimated velocities. This factor is not dependent on the true velocities, and therefore does not influence the estimation of the true velocities. The expressions for $p\left(\vec{V}^{c}\right)$ and $p\left(\vec{V}^{e} \mid \vec{V}^{c}\right)$ will be derived in the following.

An a priori knowledge is given on the true estimates, when 
the smoothness property is accepted. It has to be within a certain range relative to the neighboring velocities to fulfill the property. Applying the theory of optical flow [4] from image processing makes it possible to define a set of mathematical relations, which describes, when the transitions between velocity levels obey the smoothness property. One set of relations are the spatial and temporal derivatives. A smooth transition will be characterized by having small derivatives, as the velocity levels in neighboring locations will be fairly similar. One way of formulating the smoothness constraint is by using a Markov random field (MRF) model [4], [5], [6]. The Markov property states, that the true velocity in a given location $(x, z, t)$ depends on the velocities in a finite neighborhood of $(x, z, t)$. Implementing the derivatives as finite differences obeys the Markov property:

$$
\begin{aligned}
& \vec{v}_{d x}(x, z, t)=\vec{v}^{c}\left(x+\Delta x, z, t_{1}\right)-\vec{v}^{c}\left(x, z, t_{1}\right) \\
& \vec{v}_{d z}(x, z, t)=\vec{v}^{c}\left(x, z+\Delta z, t_{1}\right)-\vec{v}^{c}\left(x, z, t_{1}\right) \\
& \vec{v}_{d t}(x, z, t)=\vec{v}^{c}(x, z, t-\Delta t)-\vec{v}^{c}(x, z, t),
\end{aligned}
$$

where $\vec{v}_{d x}, \vec{v}_{d z}$, and $\vec{v}_{d t}$ denote the spatial and temporal derivatives, and $\Delta$ describes a temporal or spatial distance between neighbors. As an MRF model has been used, the Hammersfield-Clifford theorem [4], [7] states, that the distribution of the derivatives is a Gibbs distribution. The resulting distribution is:

$$
p\left(\vec{V}^{c}(t)\right)=\frac{1}{Z} \exp \left(-\alpha E_{1}(t)-\gamma E_{2}(t)\right),
$$

where $Z$ is a normalization factor, which assures that $\int p\left(\vec{V}^{t}\right) d x d z d t=1$. The variables $\alpha$ and $\gamma$ are scale factors, that weights the contributions from the spatial and temporal derivatives respectively. $E_{1}(t)$ and $E_{2}(t)$ are energy terms defined by:

$$
\begin{aligned}
& E_{1}(t)=\iint\left(\left\|\vec{v}_{d x}(x, z, t)\right\|^{2}+\left\|\vec{v}_{d z}(x, z, t)\right\|^{2}\right) d x d z \\
& E_{2}(t)=\iint\left\|\vec{v}_{d t}(x, z, t)\right\|^{2} d x d z .
\end{aligned}
$$

Two energy terms are used with different scaling factors to allow for the level of spatial and temporal correlation to be different. An a priori distribution has been obtained based on the a priori knowledge saying, that transitions should occur smoothly. The a priori distribution assigns a high probability to a choice of the true velocities close to the levels of the neighboring true velocities and vice versa. The chosen definition of the derivatives represent a first-order neighborhood.

The smoothness property is not the only information available. Although the velocity estimates are encumbered with uncertainty, their values give an indication of what interval of possible velocities the true velocity belongs to. This can be used to define a conditional probability distribution $p\left(\vec{V}^{e} \mid \vec{V}^{c}\right)$, which introduces a relation between the estimates and the true velocities. A relation describing a measure of error is introduced. A Gibbs distribution is usually assumed for the error distribution. The energy term is defined by:

$$
\begin{aligned}
E_{3}(t) & =\beta \iint\left(\omega_{1}\left(v_{1}^{c}(x, z, t)-v_{1}^{e}(x, z, t)\right)^{2}\right. \\
& \left.+\ldots+\omega_{k}\left(v_{k}^{c}(x, z, t)-v_{k}^{e}(x, z, t)\right)^{2}\right) d x d z
\end{aligned}
$$

where $\beta$ is a weighting parameter like $\alpha$ and $\gamma$. The index $k$ indicates the dimension of the velocity vector. The scale factor $\omega_{k}$ is introduced to account for ones faith in the velocity estimates. If the estimate is encumbered with high uncertainty, it is not a good indicator for the level of the true velocity, and its influence in the post-processing should be weighted down. When employing Kasai's autocorrelation method [8] for estimating the velocities, a measure of the variance of the estimate can be determined. In this case one could set $\omega_{k}$ proportional to the inverse of the standard deviation. The different vector velocity components might have been obtained with different estimators, and therefore $\omega$ is dependent hereof. The weighting parameters are restricted by $\alpha+\beta+\gamma=1$, and each of them take a value between 0 and 1 .

At this point a posterior, conditional probability distribution for the true velocities has been derived. An estimate of the true velocities, $\hat{v}^{c}(x, y, t)$ can be obtained by maximization of the obtained posterior distribution, $p\left(\vec{V}^{c} \mid \vec{V}^{e}\right)$, with respect to $\vec{V}^{c}$. As the posterior distribution is expressed by an exponential, the maximization of (1) equals minimization of the $\operatorname{sum} E=E_{1}+E_{2}+E_{3}$.

The energy terms are computed by integrating over the whole image, which makes the optimization very cumbersome. Besag [9] has derived a method termed the Iterated Conditional Mode (ICM) to ease the optimization. Through iterations the global posterior distribution (Eq. 1) is maximized by maximizing the local posterior distribution. Rather than working on the whole image, the maximization process is carried out on each of the pixels on an iterative basis. This approach is valid due to the Markov property. A simple filter is obtained, which computes an estimate of the true velocity, $\hat{v}^{c}$, in a given location from the values of the velocity in the neighboring locations in space and time. The velocity estimates $v^{e}$ in the neighborhood are used as initial guesses of the true velocities. In most commercial scanners only the velocity along the radial direction can be estimated. If only one iteration is performed the filter equation becomes:

$$
\begin{aligned}
\hat{v}^{c}(x, z, t)= & \frac{1}{4 \alpha+\beta+\gamma} . \\
& \left(\alpha \left(v^{e}(x-\Delta x, z, t)+v^{e}(x+\Delta x, z, t)\right.\right. \\
+ & \left.v^{e}(x, z-\Delta z, t)+v^{e}(x, z+\Delta z, t)\right) \\
+ & \gamma v^{e}(x, z, t-\Delta t) \\
+ & \left.\beta \omega v^{e}(x, z, t)\right),
\end{aligned}
$$

where the velocity vector has one component only and $\omega=1$. 
The optimal scale factors can be determined by minimization of the absolute error:

$$
\hat{\theta}=\underset{\theta}{\operatorname{argmin}} \sum_{f=1}^{F} \sum_{j=1}^{N} \sum_{i=1}^{M}\left\|v_{f}^{c}\left(x_{i}, z_{j}, t\right)-\hat{v}_{f}^{c}\left(x_{i}, z_{j}, t\right)\right\|,
$$

where $\theta$ is a vector, which consists of the scale parameters $\alpha, \beta, \gamma$. A set of $F$ 2D images are given, where the true and the estimated velocities are known. The integration has been substituted with summation, as the images consist of discrete values on a $2 \mathrm{D}$ pixel grid.

\section{Results}

As the true velocities are not known for in-vivo RF-data, simulated data resembling the in-vivo situation have been used to determine the optimal scale factors. The simulation program Field II [10], [11] was employed. It can handle any array transducer, focusing, apodization, and transducer excitation. Data resembling a carotid artery with a diameter of 6 $\mathrm{mm}$ lying at an angle of $35^{\circ}$ relative to the transducer surface are used. Tissue motion due to pulsation is included [1]. The blood motion is modeled using Womersley's pulsatile blood flow model [12]. Three seconds of data ( 3 cardiac cycles) has been simulated at a frame rate of 9 . The transducer was modeled as a $5.0 \mathrm{MHz}$ linear array. The excitation pulse was 6 cycles long.

The exact location of the vessel and the true velocities are known for these data. The blood velocity estimates, $V^{e}$, have been obtained by employing Kasai's autocorrelation estimator [8] on the RF-data.

The optimal set of scale parameters has been determined for a range of SNRs. The results are listed in Table 1.

\begin{tabular}{|c|c|c|c|c|c|c|c|}
\hline SNR & 0 & 5 & 10 & 15 & 20 & 30 & 40 \\
\hline \hline$\alpha$ & 0.77 & 0.89 & 0.80 & 0.39 & 0.22 & 0.15 & 0.13 \\
\hline$\beta$ & 0.00 & 0.00 & 0.13 & 0.59 & 0.77 & 0.85 & 0.87 \\
\hline$\gamma$ & 0.23 & 0.11 & 0.07 & 0.02 & 0.01 & 0.00 & 0.00 \\
\hline
\end{tabular}

Table 1: Optimal scale parameters $(\alpha, \beta, \gamma)$ for data with a range of SNRs $(\mathrm{dB})$.

The optimal choice of scale parameters is dependent on the SNR level. At low SNRs the filter only uses the neighboring velocities in time and space. The estimated velocities, $V^{e}$, has a high uncertainty, and therefore they should not influence the filtering much. The employed neighbors have been filtered, so the uncertainty on these have been lowered. With this in mind it seem reasonable that the neighbors influence more than the actual velocity in the given position. As the SNR increases the uncertainty on the estimate decreases. The estimated velocities are close to the truth, and therefore should play a big role in the post-processing. The spatial neighbors influences the post-processing more than the temporal neighbor. This is to be expected, as the spatial neighbors originates from the same frame, and the velocities should be very similar. The temporal correlation is dependent on frame rate. For the current scan situation the results show, that the temporal correlation is low compared to the spatial correlation due to the low frame rate. At higher frame rates one would expect an increase in the temporal correlation. The importance of the temporal and spatial neighbors decreases with increasing SNR. At high SNRs the temporal influence disappear. In practice one would never set any of the scale parameters to zero. Instead a low but non-zero value would be used.

As the true velocities are known, a measure of the performance of the filter can be obtained by computing the RMS value before and after application of the filter. Table 2 lists the RMS value before, and the improvement in \% obtained after application of the filter, stat 1 . Improvements are obtained for all SNRs and range from $15-53 \%$. The improvement is highest for low SNRs.

\begin{tabular}{|c|c|r|r|r|r|}
\hline SNR & RMS $_{\text {before }}$ & stat 1 & stat 2 & $m 1$ & $m 2$ \\
\hline \hline 0 & 0.125 & 52.6 & 46.0 & 11.0 & 27.1 \\
\hline 5 & 0.096 & 44.3 & 40.0 & 8.4 & 21.8 \\
\hline 10 & 0.076 & 34.9 & 32.4 & 5.0 & 15.2 \\
\hline 15 & 0.061 & 26.6 & 26.6 & 2.2 & 9.9 \\
\hline 20 & 0.054 & 20.6 & 21.1 & 0.0 & 6.0 \\
\hline 30 & 0.049 & 16.9 & 17.6 & -0.8 & 3.9 \\
\hline 40 & 0.048 & 15.8 & 16.5 & -0.4 & 4.1 \\
\hline
\end{tabular}

Table 2: Performance table listing the RMS error before and the improvement in \% after application of the statistical and median filters.

A choice of which set of scaling factors to use must be made, as the level of SNR is unknown a priori. Results of using the optimal scale factors for $15 \mathrm{~dB}$ on all the data sets are listed as stat 2 in Table 2. The improvement is slightly smaller than for the first case. For high SNR it seems as if the stat 2 performs better than stat 1 , which of course is not true. The reason for these results are that the performance evaluation is carried out using the RMS method, whereas the optimal scale parameters were determined by employing the absolute error. If the absolute errors were listed instead of the RMS values, one will see that the performance of stat 1 is better than stat 2 for all SNRs.

Often the post-processing is performed by applying a median filter, which acts in 1 or 2 dimensions. A comparison of performance between the statistical filter and median filters acting in 1 and 2 dimensions is carried out. The median filter acting in 1 dimension $(m 1)$ makes use of the two axial neighbors (up and down) along with the estimate it self. The second median filter $(\mathrm{m} 2)$ makes use of the 4 spatial neighbors and the estimated velocity. The performance of the median filters 
are listed in Table 2. The statistical filter performs better than the median filters for all SNRs.

Plots of the original and resulting velocity image after application of the statistical filter on the simulated data along with the true velocity image are shown in Fig. 2. A more smooth profile has been obtained, which more closely resembles the true smooth profile.

The filter was applied on in-vivo data acquired with a dedicated sampling system, which has a $30 \mathrm{MHz}$ sampling frequency and 12 bits resolution [13]. Data from the carotid artery was recorded with a $7.5 \mathrm{MHz}$ convex array. The frame rate was 18 frames/sec, and 10 seconds of data were recorded.

An example of the original and resulting CFM image is plotted in Fig. 3. The result after application of the median filter, $m 2$, is shown in the bottom plot. A certain level of smoothening is obtained with the median filter, but not to the extent as with the statistical filter, stat 2 .

Employment of the statistical filter is very simple and only requires few calculations. Memory capacity has to be set aside for storing the previous velocity image though.

\section{Conclusion}

A filter has been developed to perform the post-processing of the velocity estimates, which incorporates features of fluid flow. These features introduce restrictions on the variations allowed between the velocities in neighboring spatial and temporal locations. The scale factors in the filter varies for different levels of SNR. The filter outperforms simple median filters and is computationally very simple.

In the above it has been assumed that the same scale factors are used for all parts of the cardiac cycle. The velocity level and the level of the spatial derivatives vary over the cardiac cycle. Determination of the optimal scale factors for each frame reveals that the scale factors vary during the cardiac cycle. This aspect and its influence on the performance of the filter will be investigated in the future.

\section{Acknowledgement}

This work was supported by grant 9700883 and 9700563 from the Danish Science Foundation and by B-K Medical A/S, Gentofte, Denmark, along with grant 980018-311 from the Technical University of Denmark, Denmark. Associate Professor, Ph.D. Rasmus Larsen from the Department of Mathematical Modelling at the Technical University of Denmark is acknowledged for taking the time to discuss the theory behind optical flow and the resulting statistical methods.
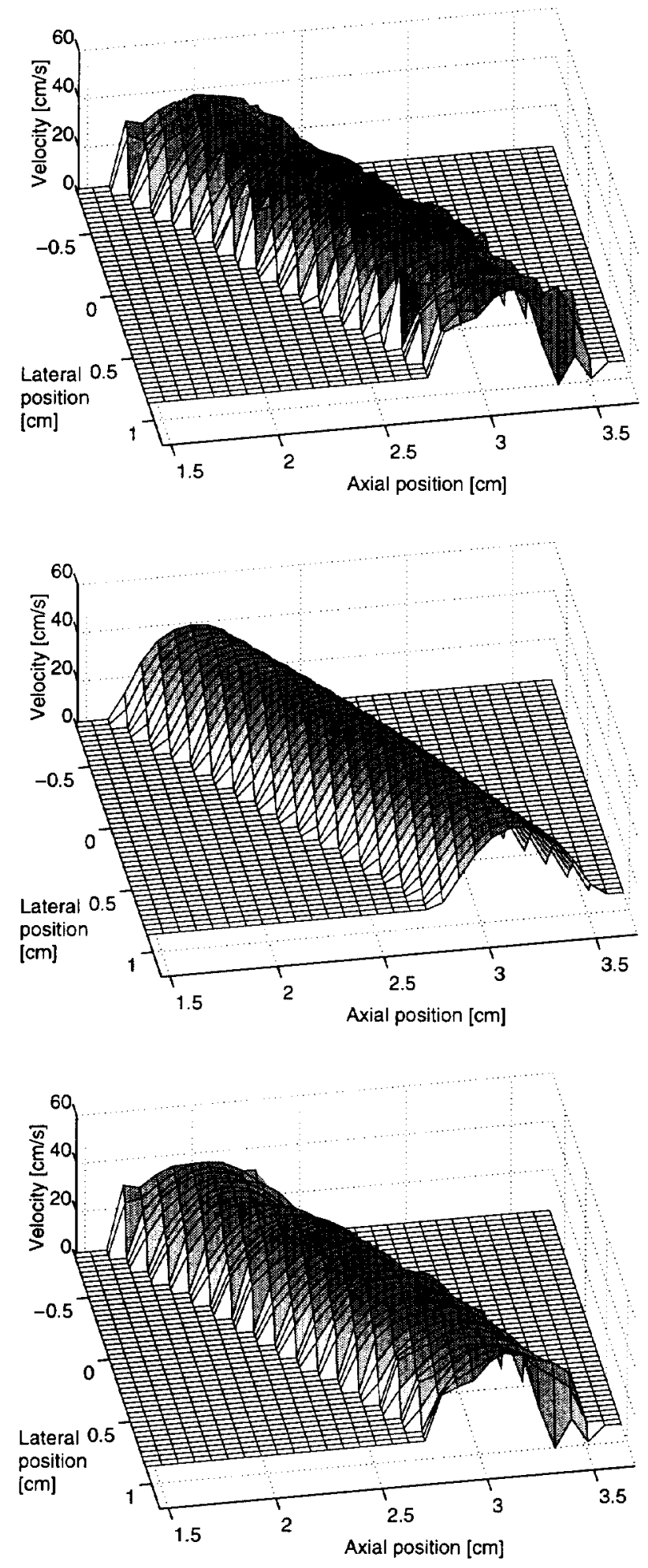

Figure 2: Plots of the velocity profile before (top) and after (bottom) application of the post-processing filter on simulated data. The middle plot shows the true velocity profile. 

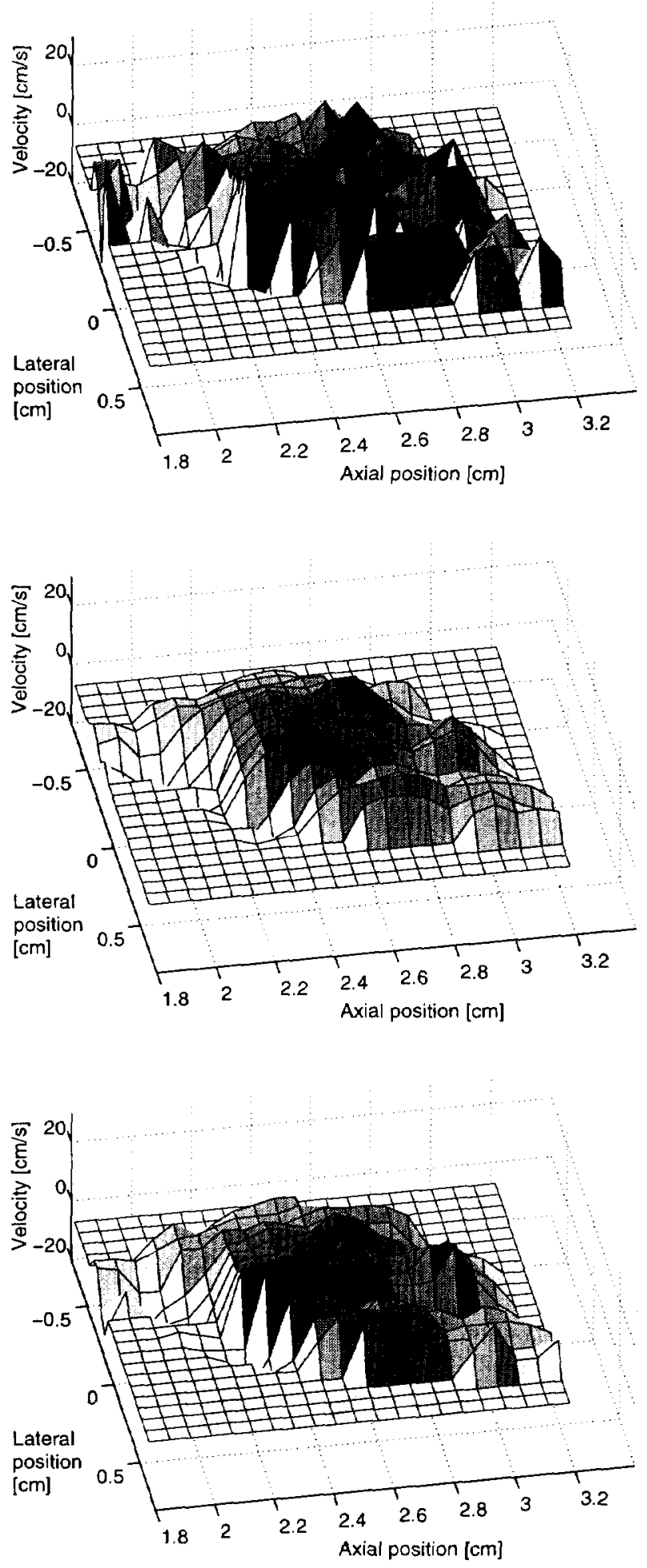

Figure 3: Plots of the velocity profile before (top) and after (middle) application of the statistical post-processing filter, stat 2 , and the application of the $m 2$ median filter (bottom) on in-vivo data.

\section{References}

[1] M. Schlaikjer, S. Torp-Pedersen, J. A. Jensen, and P. F. Stetson. Tissue motion in blood velocity estimation and its simulation. In Proc. IEEE Ultrason. Symp., pages 1495-1499, 1998.

[2] C. M. Bishop. Neural Networks for Pattern Recognition. Oxford University Press, 1997.

[3] J. Besag. Toward bayesian image analysis. J. Appl. Stat, 16:395-407, 1989.

[4] R. Larsen, K. Conradsen, and B. E. Ersbøll. Estimation of dense image flow fields in fluids. IEEE Transactions on Geoscience and Remote Sensing, 36:256-264, 1998.

[5] J. Konrad and E. Dubois. Bayesian estimation of motion vector fields. IEEE Trans. Pattern Anal. Mach. Intell., 14:910-927, 1992.

[6] S. Geman and D. Geman. Stochastic relaxation, gibbs distributions and the bayesian restoration of images. IEEE Trans. Pattern Anal. Mach. Intell., pages 721$741,1984$.

[7] R. Larsen and A. Rosholm. Motion flow detection using ICM-optimization on a massive parallel computer. Technical report, Dept. of Mathematical Modelling, Technical University of Denmark, 1994.

[8] C. Kasai, K. Namekawa, A. Koyano, and R. Omoto. Real-time two-dimensional blood flow imaging using an autocorrelation technique. IEEE Trans. Son. Ultrason., 32:458-463, 1985.

[9] J. Besag. On the statistical analysis of dirty pictures. $J$. Roy. Stat. Soc. B., 48:259-302, 1986.

[10] J. A. Jensen. Field: A program for simulating ultrasound systems. Med. Biol. Eng. Comp., 10th NordicBaltic Conference on Biomedical Imaging, Vol. 4, Supplement 1, Part 1:351-353, $1996 \mathrm{~b}$.

[11] J. A. Jensen. Users' guide for the Field II program. Technical report, Dept. of Information Technology, Technical University of Denmark, 1998.

[12] W. W. Nichols and M. F. O'Rourke. McDonald's Blood Flow in Arteries, Theoretical, Experimental and Clinical Principles. Lea \& Febiger, Philadelphia, 1990.

[13] J. A. Jensen, O. Holm, L. J. Jensen, H. Bendsen, H. M. Pedersen, K. Salomonsen, J. Hansen, and S. Nikolov. Experimental ultrasound system for real-time synthetic imaging. In Proc. IEEE Ultrason. Symp., volume 2, pages 1595-1599, 1999. 